\title{
Estudio y aplicación de indicadores turísticos en la oferta y demanda turística por periodos de tiempo en la ciudad de Cuenca - Ecuador
}

\section{Study and application of tourism indicators on tourism offer and demand by periods at Cuenca - Ecuador}

Ana Lucía Serrano ${ }^{1, *}$, Segundo Freire ${ }^{1}$, Elena Villafuerte ${ }^{1}$

\ Autor de correspondencia: ana.serrano@ucuenca.edu.ec

${ }^{1}$ Universidad de Cuenca, Facultad de Ciencias de la Hospitalidad. Tarqui y Pío Bravo. Cuenca 010107. Ecuador.

Artículo original de la ponencia presentada en el IV CONGRETUR - "El impacto del turismo en el desarrollo del Ecuador", Universidad Central del Ecuador, 21 al 24 de noviembre de 2018

\begin{abstract}
Resumen
El estudio presentado está basado en el proyecto "Análisis de los indicadores de sostenibilidad y competitividad turística con incidencia en las estrategias de gestión sostenible, gobernanza y políticas públicas del cantón Cuenca", en el cual se ha planteado un análisis de variables en la oferta, tales como plazas disponibles, noches de hospedaje, segmento de huésped y ocupación hotelera; mientras que en el caso de la demanda se analizan indicadores, tales como comportamiento del visitante nacional y extranjero, aspectos sociodemográficos y lugares mayormente visitados. Se plantea una metodología cuantitativa descriptiva y como herramienta para el levantamiento de información se formulan encuestas acordes a las variables de la oferta y demanda turística, obteniendo en la oferta una respuesta promedio de $95,2 \%$, mientras que en la demanda se analizan 46 variables con $94,6 \%$ de respuesta.
\end{abstract}

Palabras clave: Indicadores, Oferta, Demanda, Turistas y Hoteles, Cuenca.

\begin{abstract}
This study is based on the project "Analysis of indicators of sustainability and tourism competitiveness with an impact on the strategies of sustainable management, governance and public policies of the Cuenca canton", in which an analysis of variables in the tourist offer such as: available places, nights of lodging, guest segment and hotel occupancy, while in the case of demand, indicators such as domestic and foreign visitors' behavior, socio-demographic aspects, and most visited places are analyzed. It is proposed a descriptive quantitative methodology and a tool for the collection of information, surveys are designed according to the variables of the tourist offer and demand, obtaining in the offer indicator an average response of $95.2 \%$, while in the demand one 46 variables are analyzed with $94.6 \%$ responses.
\end{abstract}

Keywords: Indicators; Offer; Demand; Tourists and Hotels; Cuenca, Ecuador. 


\section{Introducción}

Cuenca es conocida como una ciudad turística por poseer aspectos que convierten en única la visita de un turista. Ofrece una riqueza arquitectónica y cultural, espacios naturales, un centro histórico con aires coloniales y construcciones republicanas, vestigios arqueológicos y tradiciones; además, en el año1999 fue declarada Patrimonio de la Humanidad (Ministerio de Turismo, 2014). La ciudad de Cuenca cuenta con 220 establecimientos de alojamiento, distribuidos así: $56 \%$ de hostales, $34 \%$ de hoteles, $4 \%$ de hoteles boutique, $3 \%$ de hosterías y $2 \%$ de apartamentos turísticos y otras modalidades de alojamiento; además, existe una oferta de 8.534 plazas diarias (Serrano \& Villafuerte, 2017, p. 61).

Existe déficit de indicadores locales para la administración del turismo. Los datos agregados, por país, limitan la toma de decisiones para las empresas de la ciudad de Cuenca. Por esta razón se propuso iniciar con un proyecto de creación de indicadores turísticos denominado "Análisis de los indicadores de sostenibilidad y competitividad turística con incidencia en las estrategias de gestión sostenible, gobernanza y políticas públicas del cantón Cuenca", con base en la oferta y demanda turística, la que serviría como fuente de consulta académica y de apoyo a la gestión de procesos de la planta turística, entre julio de 2015 y diciembre de 2016.

La oferta turística se refiere a la integración de servicios que suministra elementos de la planta turística y componentes no turísticos (Boullón, 2006). Mientras que la demanda turística se refiere al número de turistas que concurren a una región, centro o atractivo turístico, los ingresos que producen para el país y área receptora y su contribución entre distintos servicios ocupados (Boullón, 2006, pp. 32-33).

El objetivo del estudio es crear y analizar un sistema de indicadores turísticos del cantón Cuenca, a través de la recopilación de información primaria y secundaria para su posterior implementación y manejo a fin de caracterizar el mercado turístico local.

La implementación del sistema de indicadores turísticos revela un crecimiento en el mercado turístico local y tiene una relación directamente proporcional con la evolución del mercado turístico nacional e internacional, se preveía que la demanda turística en la ciudad de Cuenca aumentaría en un 15\%; por otro lado, la oferta turística crecería en un 5\%. El conocimiento de los indicadores de competitividad y sostenibilidad turística locales ha generado información para los agregados estadísticos nacionales. De esta forma, desde lo local a lo nacional, promovería propuestas de gobernanza, políticas públicas y estrategias de gestión sostenible.

La Organización Mundial de Turismo registró 1.418.159 llegadas de extranjeros para el año 2016, disminuyendo la cifra de ingresos al país en $8,18 \%$ con relación al año anterior. Se estimó también que el $69 \%$ de llegadas de extranjeros al Ecuador están motivadas por turismo (Ministerio de Turismo, 2016, p. 9). El turismo para el mundo representa un sector que crece en importancia, y depende del impulso que los países promuevan en sus propuestas estratégicas de largo plazo. Para países como España, Ecuador, Estados Unidos, China, crece su contribución a su Producto Interno Bruto. Para el año 2016 ya el turismo representó el 1.8\% del PIB mundial y $6.8 \%$ de sus exportaciones (Serrano, Freire, Espinoza, Andrade \& Villafuerte, 2018).

El análisis de indicadores aporta con datos específicos de gestión, como por ejemplo, el promedio de noches de alojamiento, la tendencia que ocupación de la oferta total de plazas, el tipo de clientes que ocupan la oferta turística, el impacto real de los mayoristas de turismo o los intermediarios para captar cierto tipo de clientes. Desde la perspectiva de la demanda, conocer el tipo de establecimientos que ocupan, respecto de su categoría, aportaría a la construcción de perfiles de negocio, tanto con metodologías aleatorias como descriptivas. Los perfiles de tipo aleatorio y con escenarios probabilísticos con un error estadístico igual o inferior al 5\% contribuirían a la especialización de la planta turística, a su dedicada actividad de mercadotecnia y al desarrollo de productos específicos. Los indicadores descritos fueron elaborados a partir del proyecto en mención y retomados por el presente estudio para su difusión en un Boletín de Indicadores Turísticos del cantón Cuenca (Serrano, Freire, Sanmartín, Espinoza, Farfán, Andrade \& Villafuerte, 2018).

\section{Materiales y métodos}

La metodología empleada es de tipo descriptivo-cuantitativo. Para el levantamiento de información se utilizaron encuestas estructuradas con variables, tales como disponibilidad de plazas, número de noches de hospedaje, procedencia de los huéspedes, segmento de huésped, modalidad de empleo, necesidades de capacitación y formación del sector hotelero. Se aplicó la fórmula muestral al universo de establecimientos de alojamiento del año 2015 y se aplicó de manera voluntaria (no aleatoria) en relación con la disponibilidad de cooperación de los establecimientos que aceptaron ingresar al estudio. Se levantó información de 119 establecimientos, entre hoteles, hoteles boutique, hostales, hosterías y pensiones, y casas de 
huéspedes, entre julio del año 2015 y diciembre de 2016, con una tasa de respuesta de $95,2 \%$.

Con respecto al estudio de demanda, se realizó una segmentación de los lugares más visitados dando un total de nueve conglomerados y de esta manera las encuestas fueron tomadas de manera aleatoria; se considera un índice de error del 5,4\% y el 94,6\% de asertividad en la respuesta. Las bases de datos tanto de la oferta como de la demanda turística fueron procesadas en el programa SPSS y el procesamiento de información con el programa Tableau Public.

\section{Resultados}

\subsection{Oferta turística}

Con base en el catastro de alojamiento del año 2015 se realiza el cálculo del número de plazas ofertadas en los establecimientos de hospedaje, dando como resultado total 8.534 , distribuidas por categorías, así: lujo, primera, segunda, tercera y cuarta, como se puede visualizar en la tabla 1.

Tabla 1. Número de plazas en los establecimientos de alojamiento por categoría

\begin{tabular}{lcc}
\multicolumn{1}{c}{ Categoría } & Número de registros & Número de plazas de hospedaje \\
\hline Lujo & 2 & 218 \\
\hline Primera & 67 & 3.137 \\
\hline Segunda & 81 & 2.950 \\
\hline Tercera & 69 & 2.195 \\
\hline Cuarta & 1 & 34 \\
\hline Total General & $\mathbf{2 2 0}$ & $\mathbf{8 . 5 3 4}$ \\
\hline
\end{tabular}

Nota: Elaboración propia a partir del Catastro de Alojamiento. Ministerio de Turismo (2016).

En el caso del número de noches en las que pernocta un huésped se detectó que depende de la categoría del establecimiento, pero de manera general se presenta como mínimo una noche y máximo tres, tal como se puede observar en la tabla 2.

Tabla 2. Número de noches de alojamiento de acuerdo a la categoría

\begin{tabular}{|c|c|c|c|c|c|c|}
\hline Año & Mes & Lujo & Primera & Segunda & Tercera & Cuarta \\
\hline \multirow{6}{*}{2015} & Julio & 1,8 & 2,7 & 2,4 & 3,2 & 3,6 \\
\hline & Agosto & 2,1 & 2,9 & 2,3 & 2,9 & 1,6 \\
\hline & Septiembre & 1,5 & 3 & 2,3 & 2,7 & 3,6 \\
\hline & Octubre & 1,5 & 1,4 & 1,5 & 1,7 & \\
\hline & Noviembre & 2 & 1,4 & 1,8 & 1,9 & \\
\hline & Diciembre & 2 & 1,5 & 1,4 & 1,7 & \\
\hline \multirow{12}{*}{2016} & Enero & 1,5 & 1,6 & 1,8 & 1,5 & \\
\hline & Febrero & 1,7 & 1,9 & 1,9 & 1,4 & \\
\hline & Marzo & 1,8 & 1,7 & 1,9 & 1,3 & \\
\hline & Abril & 1 & 1,9 & 2,4 & 2 & 2 \\
\hline & Mayo & 1,4 & 1,9 & 2 & 1,7 & 1,3 \\
\hline & Junio & 1,7 & 1,5 & 1,1 & 1,2 & 2 \\
\hline & Julio & 1 & 1 & 1 & 1 & 1 \\
\hline & Agosto & 1 & 0,9 & 0,9 & 0,9 & 1 \\
\hline & Septiembre & 1,5 & 1,7 & 1,7 & 1,7 & 1,6 \\
\hline & Octubre & 1,5 & 1,7 & 1,7 & 2,1 & 1,4 \\
\hline & Noviembre & 1,7 & 2,2 & 1,9 & 1,9 & 1,6 \\
\hline & Diciembre & 1,5 & 1,9 & 2 & 1,6 & 1,2 \\
\hline
\end{tabular}

Nota: Elaboración propia a partir de las encuestas de la oferta turística años 2015 y 2016. 
Con respecto al segmento de huéspedes que se alojan en los diferentes establecimientos de acuerdo a la ocupación hotelera, en el cuarto trimestre del año 2015 con un $45 \%$ se identificó a los huéspedes con reserva, seguido del corporativo y turista con el $38 \%$, mientras que en el año 2016 el segmento pre- dominante son las agencias de viajes con el $80 \%$, seguido de los grupos con el $47 \%$, como se puede visualizar en la tabla 3. La información del año 2015 se presenta desde el cuarto trimestre, por el hecho de que el primer levantamiento de información se generó a partir de julio del año en mención.

Tabla 3. Ocupación de los establecimientos de alojamiento por segmento de mercado

Año

Segmento

Trimestre 1

Trimestre 2

Trimestre 3

Trimestre 4

\begin{tabular}{|c|c|c|c|c|c|}
\hline & Agencias de viaje & & & & $33 \%$ \\
\hline & Comerciantes & & & $14 \%$ & $10 \%$ \\
\hline & Corporativo & & & $38 \%$ & $35 \%$ \\
\hline \multirow[t]{8}{*}{2015} & Grupos & & & $23 \%$ & \\
\hline & Huésped con reserva & & & $22 \%$ & $45 \%$ \\
\hline & Huésped sin reserva & & & $21 \%$ & $21 \%$ \\
\hline & Turista & & & $26 \%$ & $38 \%$ \\
\hline & Agencias de viaje & & $80 \%$ & & \\
\hline & Comerciantes & $7 \%$ & $7 \%$ & & \\
\hline & Corporativo & $29 \%$ & $20 \%$ & $26 \%$ & $28 \%$ \\
\hline & Grupos & & & & $47 \%$ \\
\hline \multicolumn{6}{|l|}{2016} \\
\hline & Huésped con reserva & $7 \%$ & & & \\
\hline & Huésped sin reserva & $18 \%$ & $17 \%$ & $16 \%$ & $20 \%$ \\
\hline & Turista & $29 \%$ & $24 \%$ & $27 \%$ & $34 \%$ \\
\hline & Todos & & & & $46 \%$ \\
\hline
\end{tabular}

Nota: Elaboración propia con base en las encuestas de la oferta turística año 2015 y 2016.

En la ocupación hotelera de la ciudad de Cuenca en el año 2016, se destaca que la mayor ocupación promedio de los establecimientos de lujo fue en el mes de noviembre, siendo esta de $65 \%$; en los de primera categoría en el mes referido la ocupación fue del 57\%; en los de segunda en el mes en mención se sitúa la ocupación en el 36\%; en tanto que en los establecimientos de tercera, en el mes de noviembre, se presenta una ocupación promedio del $37 \%$; finalmente, en los de cuarta categoría se presenta en el mes de junio un $50 \%$ de ocupación hotelera, como se puede observar en la figura 1. 


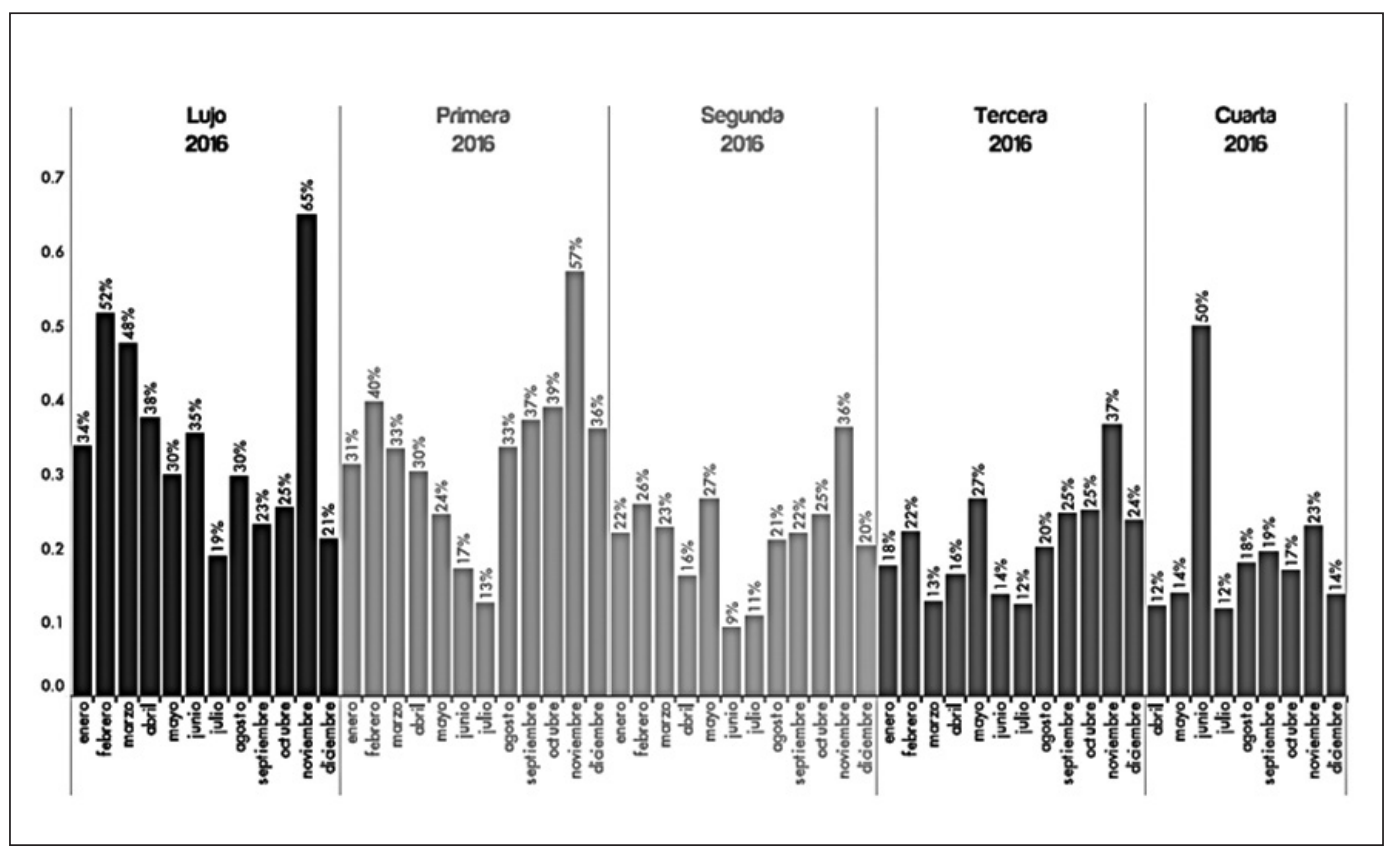

Figura 1. Ocupación hotelera en el año 2016. Elaboración propia a partir de las encuestas de la oferta turística, años 2015 y 2016.

\subsection{Demanda turística}

En los años 2015 y 2016 la ciudad de Cuenca recibió turistas nacionales de diferentes destinos del
Ecuador. En mayor proporción, con el 36,25\%, proceden de la ciudad de Guayaquil, luego está Quito con un $28,51 \%$ de visitantes, como se puede visualizar en la tabla 4.

Tabla 4. Procedencia de los visitantes de las ciudades del Ecuador

\begin{tabular}{cc} 
Procedencia & Total \\
\hline Ambato & $4,07 \%$ \\
\hline Esmeraldas & $1,22 \%$ \\
\hline Guayaquil & $36,25 \%$ \\
\hline Loja & $10,18 \%$ \\
\hline Macas & $0,81 \%$ \\
\hline Machala & $11,81 \%$ \\
\hline Manta & $1,83 \%$ \\
\hline Pasaje & $0,81 \%$ \\
\hline Portoviejo & $2,85 \%$ \\
\hline Quito & $28,51 \%$ \\
\hline Riobamba & $1,63 \%$
\end{tabular}

Nota: Elaboración propia a partir de las encuestas de la oferta turística años 2015 y 2016.

En cuanto a los turistas extranjeros que visitan la ciudad de Cuenca en el año 2015, en su mayoría, con un $40 \%$, proceden de Estados Unidos, seguidos de visitantes de Argentina con el 11,84\%. En el caso del año 2016 la realidad es otra pese a los eventos ocurridos en el Ecuador en este año, se tiene que un $51,59 \%$ de turistas de Estados Unidos visitaron la ciudad de Cuenca, en comparación con el año anterior 
se obtiene un incremento del $11,59 \%$ y en el caso de los visitantes de Argentina disminuyen al 9,55\% en este año, es decir, un 2,29\% menos con relación al año 2015, tal como se puede observar en la tabla 5.

Tabla 5. Procedencia de los turistas en los años 2015 y 2016

\begin{tabular}{ccc} 
Procedencia & Año 2015 & Año 2016 \\
\hline Estados Unidos & $40 \%$ & $51,59 \%$ \\
\hline Argentina & $11,84 \%$ & $9,55 \%$ \\
\hline Canadá & $11,02 \%$ & $7,39 \%$ \\
\hline Colombia & $9,39 \%$ & $7,90 \%$ \\
\hline Alemania & $8,57 \%$ & $5,35 \%$ \\
\hline España & $4,49 \%$ & $2,80 \%$ \\
\hline Perú & $4,49 \%$ & $1,91 \%$ \\
\hline Chile & $3,27 \%$ & $1,91 \%$ \\
\hline Francia & $2,45 \%$ & $3,82 \%$ \\
\hline Italia & $2,04 \%$ & $1,66 \%$ \\
\hline Inglaterra & $1,63 \%$ & $4,33 \%$ \\
\hline Suiza & $0,82 \%$ & $1,78 \%$ \\
\hline
\end{tabular}

Nota: Elaboración propia a partir de las encuestas de la oferta turística año 2015 y 2016.

En el objetivo de visita en el año 2015 en el cuarto trimestre, el 74,30\% de ecuatorianos que visitan la ciudad de Cuenca lo hacen por vacaciones, recreación y ocio, el 59,80\% de extranjeros estadounidenses también llegan a la ciudad por la razón mencionada anteriormente. Mientras que en el año 2016, en el primer trimestre, el 48,89\% de ecuatorianos llegan a Cuenca por vacaciones, recreación y ocio, de la misma manera el 55,32\% de estadounidenses y en el segundo trimestre del año 2016, tanto ecuatorianos como estadounidenses mantienen su objetivo de visita por vacaciones, recreación y ocio. Es decir que en los años 2015 y 2016 los otros objetivos planteados no poseen una alta prioridad, prácticamente se encuentran en un segundo plano, como se visualiza en la figura 2.

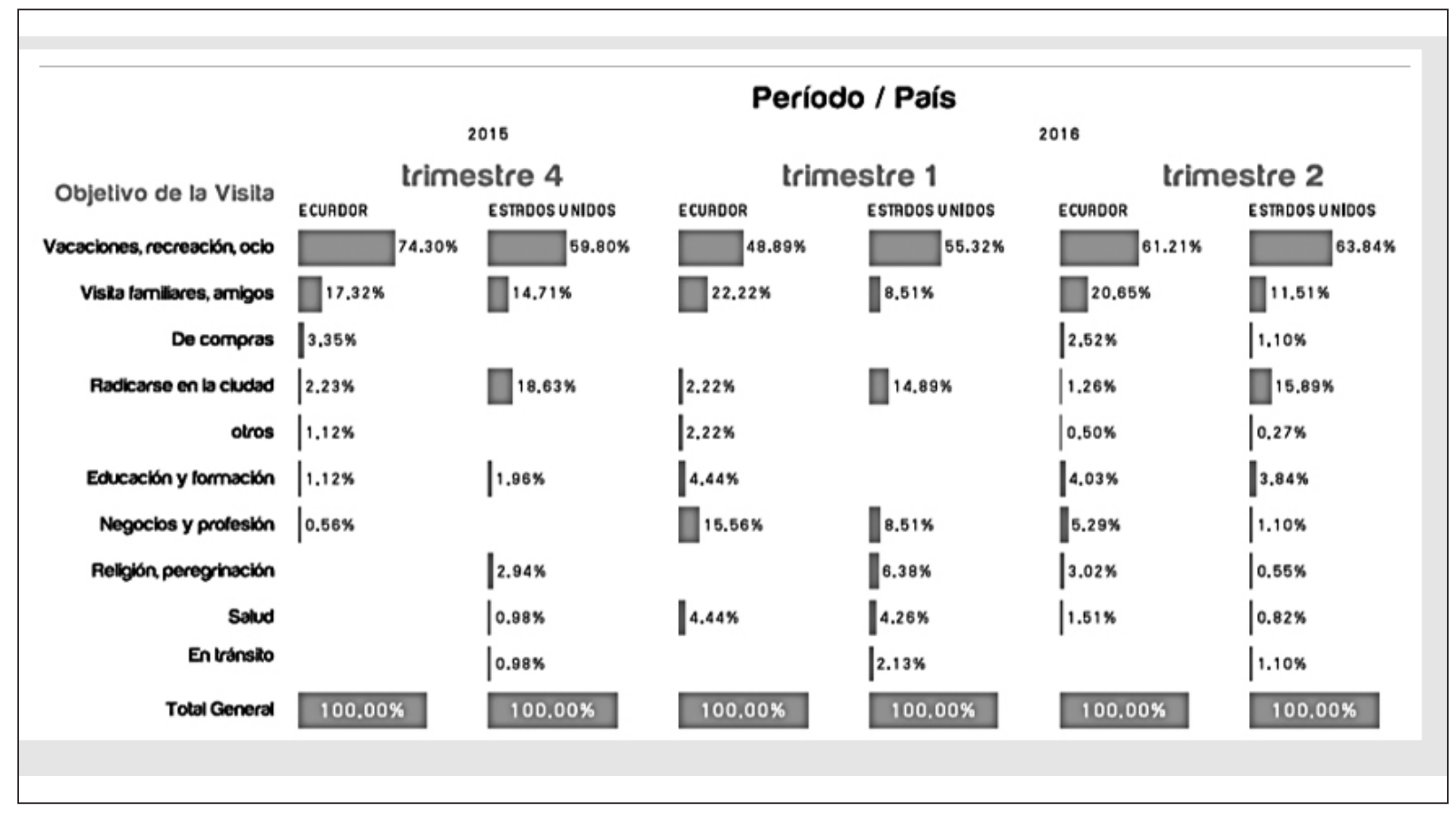

Figura 2. Objetivo de la visita a la ciudad de Cuenca. Elaboración propia a partir de las encuestas de la oferta turística, años 2015 y 2016. 
La preferencia de hospedaje varía de acuerdo al rango de edad, el $33 \%$ de huéspedes de 15 a 25 años prefieren hospedarse en casas de amigos o familiares, mientras que el $36 \%$ de 26 a 35 años de edad optan por alojarse en hostales. Por otro lado, el 37\% de huéspedes de 36 a 45 años eligen quedarse en hoteles, en el caso del $40 \%$ de los visitantes de 46 a 55 años seleccionan como alojamiento los hoteles, en tanto que el $32 \%$ de personas de 56 a 65 años escogen como alojamiento también los hoteles. Finalmente, el 29\% de huéspedes de más de 66 años gustan de hospedarse en casas de amigos o familiares, los datos expuestos pueden ser observados en la tabla 6 .

Tabla 6. Preferencia de alojamiento por grupo de edad de los visitantes

\begin{tabular}{|c|c|c|c|c|c|c|}
\hline \multirow{2}{*}{ Alojamiento } & \multicolumn{6}{|c|}{ Edad } \\
\hline & 15 a 25 años & 26 a 35 años & 36 a 45 años & 46 a 55 años & 56 a 65 años & Más de 66 años \\
\hline Airbnb & & & $0,30 \%$ & & & $2 \%$ \\
\hline Apartamento & $1 \%$ & $1 \%$ & $0,30 \%$ & $1 \%$ & $5 \%$ & $17 \%$ \\
\hline Casa de amigos / familiares & $33 \%$ & $20 \%$ & $26 \%$ & $23 \%$ & $24 \%$ & $29 \%$ \\
\hline Casa de huéspedes & $16 \%$ & $13 \%$ & $13 \%$ & $14 \%$ & $17 \%$ & $15 \%$ \\
\hline Casa rentada & $0,20 \%$ & $0,40 \%$ & $1 \%$ & $2 \%$ & $1 \%$ & \\
\hline Departamento en renta & $0,20 \%$ & $0,20 \%$ & & $2 \%$ & $2 \%$ & \\
\hline Hostal & $29 \%$ & $36 \%$ & $19 \%$ & $16 \%$ & $17 \%$ & $15 \%$ \\
\hline Hostería & & & & $1 \%$ & & \\
\hline Hotel & $19 \%$ & $28 \%$ & $37 \%$ & $40 \%$ & $32 \%$ & $19 \%$ \\
\hline Hotel Boutique & $1 \%$ & $1 \%$ & $4 \%$ & $2 \%$ & $2 \%$ & $3 \%$ \\
\hline
\end{tabular}

Nota: Elaboración propia a partir de las encuestas de la oferta turística, año 2016.

Entre los lugares más visitados en la ciudad de Cuenca durante su permanencia se tiene que en el año 2015 el $62,37 \%$ de turistas asistieron a los museos mientras que en el 2016 se da un incremento de visitantes del 6,98; seguidamente en el 2015 con el $14,55 \%$ lo más frecuentado fueron los eventos cul- turales, situación que no ocurre en el año 2016 pues se presenta una disminución del 7,10\% de visitantes. Otro de los lugares de visita en el 2015 con un $10,19 \%$ es la plaza de las flores, mientras que en el año 2016 sucede un leve crecimiento de un $0,35 \%$, como se puede visualizar en la tabla 7 .

Tabla 7. Lugares visitados por los turistas que llegan a Cuenca

\begin{tabular}{lcc} 
& $\mathbf{2 0 1 5}$ & $\mathbf{2 0 1 6}$ \\
\hline Museos & $62,37 \%$ & $69,35 \%$ \\
\hline Eventos culturales & $14,55 \%$ & $7,45 \%$ \\
\hline Plaza de las flores & $10,19 \%$ & $10,54 \%$ \\
\hline El Cajas & 9,15 & $7,30 \%$ \\
\hline Iglesias & $2,49 \%$ & $3,61 \%$ \\
\hline Centro histórico & $0,62 \%$ & $0,53 \%$ \\
\hline Fiestas tradicionales & $0,42 \%$ & $0,68 \%$ \\
Baños & $0,21 \%$ & $0,53 \%$
\end{tabular}

Nota: Elaboración propia a partir de las encuestas de la oferta turística, año 2016. 


\section{Discusión}

Generar indicadores turísticos es de importancia, y más al tratarse de una ciudad que se considera como la tercera más importante del Ecuador, pues es necesario analizar diferentes variables que se vinculan al turismo, tales como capacidad de 'carga' en los establecimientos de alojamiento, preferencias de hospedaje acorde a la edad, objetivo de la visita, lugares que son frecuentados mayormente, procedencia de los turistas, ocupación hotelera de acuerdo al segmento de mercado, ocupación hotelera acorde a las categorías de establecimientos de alojamiento y número de noches que se hospedan los huéspedes, por el hecho que todos estos indicadores van a generar importantes aportes de información para el sector turístico como para los actores involucrados tanto internos como externos. $\mathrm{Al}$ iniciar esta investigación se analizaron las necesidades del sector para encaminar las decisiones de estudio y la intervención de la academia.

Analizados los indicadores de oferta turística, se obtiene como resultado preliminar que el sector turístico de Cuenca, posee una capacidad de 8.534 plazas de alojamiento diarias indicando que esta ciudad está preparada para recibir una carga importante de huéspedes, pero al analizar la realidad de la ocupación hotelera se pudo observar que no se cubre en su totalidad la capacidad ofertada. En el año 2016 el mes de mayor ocupación fue noviembre en los establecimientos de las categorías de lujo, primera, segunda y tercera, mientras que en los de cuarta el mayor porcentaje se presenta en el mes de junio. Se puede interpretar que el mes de noviembre, por ser feriado, fue el que mejor resultados proporcionó a los establecimientos de alojamiento; el segmento de huéspedes que se aloja en el caso del año 2015 con el $45 \%$ hace referencia al huésped con reserva, seguido de turistas y corporativo, mientras que el 2016 el segmento predominante son las agencias de viaje con el $80 \%$ y seguidamente grupos con el $47 \%$. Es importante indicar que en este año se buscó la forma de vender el destino Cuenca, pese que esta ciudad no se vio afectada en su infraestructura por un desastre natural como ocurrió con otras ciudades del Ecuador, se tenía la necesidad de fomentar la ocupación hotelera, fue ese el momento en que las agencias de viajes intervinieron.

En la demanda turística con respecto a la procedencia de visitantes nacionales, se obtiene que turistas de las ciudades de Guayaquil y Quito son quienes con mayor frecuencia visitan Cuenca, el dato proporcionado permitirá establecer gestiones de mercadeo para enfocar la dirección de los esfuerzos. Mien- tras que en el caso de los turistas extranjeros tanto en 2015 como en 2016 proceden de Estados Unidos y Argentina, es decir, se tiene un panorama claro para enfocar la gestión de promoción. En el objetivo de la visita se presenta que en 2015 y 2016 tanto nacionales como extranjeros, en su mayoría, asisten por vacaciones, recreación y ocio, es decir, que los turistas buscan salir de la rutina para enfocarse netamente al descanso. En el caso de la preferencia de alojamiento de acuerdo al rango de edad este va a variar, en el estudio se detecta que los establecimientos de mayor frecuencia son hostales y hoteles y, en casos particulares, las casas de amigos o familiares. Y finalmente, los turistas que han llegado a la ciudad de Cuenca en mayor cantidad han visitado los museos, lo cual aclara el panorama del objetivo de visita.

\section{Conclusiones}

En este artículo se aborda brevemente la importancia de generar indicadores turísticos por el hecho de que se trata de herramientas que son de vital importancia para la planificación del destino, también para identificar y crear un perfil adecuado que permita establecer cuál es el consumo que se realiza de nuestro producto turístico, tal como es el caso de la ciudad patrimonial de Cuenca - Ecuador.

Al realizar el análisis de los datos presentados en el estudio se determina que se puede establecer un termómetro desde la perspectiva del hospedaje para medir cuántos turistas llegan a Cuenca, también se define quiénes visitan y quiénes son turistas en la ciudad, además precisa cuál es la disponibilidad o capacidad de 'carga' que posee Cuenca, es decir, cuántas personas pueden quedarse en los establecimientos de alojamiento y el comportamiento del turista a la hora de realizar actividades vinculadas al turismo.

Se manifiesta que se puede generar soluciones a las interrogantes turísticas que presenta la ciudad de Cuenca en el ámbito del turismo, abordando y cubriendo las necesidades de conocimiento de este sector, el mismo que en los últimos años se ha visto afectado. Con la información generada se pretende buscar nuevas soluciones para mejorar la economía de este sector, además se intenta vincular a la academia para que realice investigación en relación a la situación que se vive en el turismo de la ciudad de Cuenca.

Con la ejecución de la investigación relacionada a la planta del sector turístico cuencano se pretende contribuir con información sobre los impactos que están causando los diferentes cambios sociales, políticos, ambientales, económicos, entre otros, y los 
efectos tanto directos como indirectos a los diferentes actores, por tal motivo se considera este análisis

\section{Referencias}

Boullón, R. (2006). Planificación del espacio turístico. México: Trillas.

Serrano, A. L., Freire, S., Sanmartín, I., Espinoza, F., Farfán, K., Andrade, S., \& Villafuerte, E. (2018). Boletín de indicadores turísticos del cantón Cuenca. Cuenca: Departamento de Investigación de la Facultad de Ciencias de la Hospitalidad.

Brida, J. G., Pereyra, J. S., Such Devesa, M. J., \& Zapata Aguirre, S. (2008). La contribución del turismo al crecimiento económico. Cuadernos de Turismo, 22, 35-46.

Serrano, A. L., \& Villafuerte Pucha, E. (2017). Indicadores turísticos: oferta y demanda de la ciudad como parte preliminar de un aporte a la situación turística que se vive en la ciudad de Cuenca.

patrimonial de Cuenca - Ecuador. Revista Latinoamericana de Turismología, 3(1), 58-68.

Serrano, A. L., Freire, S., Espinoza, F., Andrade, S., \& Villafuerte, E. (2018). Modeling of Tourist Profiles with Decision Trees in a World Heritage City: The Case of Cuenca (Ecuador). Tourism Planning \& Development, 1-21. https://doi.org/10.1080/ 21568316.2018.1501731

Ministerio de Turismo. (2016). Boletín de Estadísticas Turisticas 2012-2016. Quito: Ministerio de Turismo.

Ministerio de Turismo. (2016). Catastro Nacional de Alojamiento. Cuenca, Azuay, Ecuador. 